\title{
Post Exercise Desaturation Can Help Identifying Treatment Responders of COPD Pulmonary Hypertension in Real World: An Appraisal
}

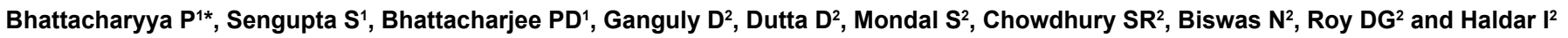
${ }^{1}$ Institute of Pulmocare and Research, Kolkata, India

${ }^{2}$ Kolkata-Ph group, BM Birla Heart Research Centre, Kolkata, India

\begin{abstract}
Background: COPD associated pulmonary hypertension (COPD-PH) leads to desaturation on exercise. It affects the health status and survival adversely.

Objective: To look for the change in pulse rate, arterial oxygen saturation $\left(\mathrm{SpO}_{2}\right)$, and the health status on treatment of COPD-PH with sildenafil administered on a desaturation criteria in response to a simple exercise protocol.

Methods: Patients of COPD-PH (diagnosed clinic-radio-echocardiographically) were subjected to a simple and uniform exercise test involving sitting and moving between two chairs ( 2 chair test). The baseline and post exercise measurement of the pulse rate and $\mathrm{SpO}_{2}$ at every 10 seconds were recorded for 120 seconds. Patients showing a desaturation $\geq 3 \%$ ware prescribed $\mathrm{PH}$-specific pharmacotherapy as sildenafil. Change in resting pulse rate, $\mathrm{SpO}_{2}$, and COPD assessment test (CAT score) were noted and compared after $90 \pm 15$ days of observation with or without sildenafil.
\end{abstract}

Results: Out of 147 COPD patients, $89(60.5 \%)$ had PH of which 62 subject qualified for sildenafil. At the end of follow up 57 and 27 subjects (with or without receiving sildenafil) were available for statistical calculation. Both the group of patients were similar in age, FEV1, FVC, and FEV1/FVC. The treated group showed an improvement in CAT $(P<0.0001)$ and saturation $(p=0.0003)$ while those on COPD therapy alone had mild worsening in arterial oxygen saturation $(p=0.03)$.

Inference: Patients of COPD-PH treated on the basis of exercise desaturation showed significant improvement to $\mathrm{PH}$ specific pharmacotherapy. The observation demands attention and validation to define the indication of $\mathrm{PH}$ specific pharmacotherapy in COPD-PH.

Keywords: COPD; Pulmonary circulation; Pulmonary hypertension; CAT; Sildenafil

Abbreviations: COPD: Chronic Obstructive Pulmonary Disease; PH: Pulmonary Hypertension; FEV1: Forced Expiratory Volume In 1 Second; FVC: Forced Vital Capacity; $\mathrm{SpO}_{2}$ : Arterial Oxygen Saturation; 2CT: Two Chair Test; 6MWT: 6 Minutes Walk Test; RHC: Right Heart Catheterization.

\section{Introduction}

COPD is a common airway disease and is a growing public health problem [1]. By 2020, it is predicted to be the third leading cause of death $[2,3]$. The sufferers of COPD often develop pulmonary hypertension $(\mathrm{PH})$ that adds to the morbidity and affects the survival adversely [4]. According to recent studies, the prevalence of $\mathrm{PH}$ in COPD has been reportedly to be approximately $30 \%-70 \%$ [5]. The majority of this problem is encountered in advanced COPD patients with mild to moderate increase in pulmonary artery pressure (PAP) and is referred to as COPD-PH. However, a small number of patients show high pulmonary arterial pressure (PAP) with relatively mild airflow limitation (expressed as PH-COPD) [6]. While the guidelines are categorically instructive regarding administering $\mathrm{PH}$-specific treatment in PH-COPD patients, they are silent regarding the majority (COPD-PH cases) possibly for lack of high quality evidence to define pharmacotherapy in them. This lack of directives in guidelines might have led to a deterrent effect on decision for $\mathrm{PH}$ specific pharmacotherapy by a physician for COPD-PH. Furthermore, for the diagnosis of $\mathrm{PH}$, the situation is often complicated by the demand of right heart catheterization (RHC) which is often not possible in the vast majority of the real world. Thus, a huge number of sufferers of COPD$\mathrm{PH}$ may remain deprived of the benefits of anti PH medications.

In this article, the authors have circumvented the need of RHC by a well-defined clinic-radio-echocardiographic criterion and have tried to count the extent of desaturation following a simple and defined exercise test as an indication of $\mathrm{PH}$ pharmacotherapy. They have observed the response to anti-PH based on the change in post exercise desaturation and health status.

\section{Methods}

The work had been done at the Institute of Pulmocare and Research based on an ethically approved protocol (approved by Institutional ethics committee of Institute of Pulmocare and Research) (CTRI numberCTRI/2015/07/005962). The subjects were screened and recruited from the out-patient department of the Institute. We formed a "Kolkata PH group" the members of which remained involved in framing the major strategies and the guideline for treatment. The protocol included the following steps:

\section{Diagnosis of COPD}

The COPD subjects were diagnosed clinicoradiologically and

*Corresponding author: Parthasarathi Bhattacharyya, Institute of Pulmocare and Research, Kolkata, India, Tel: 9165481305; E-mail: parthachest@yahoo.com

Received July 06, 2018; Accepted August 31, 2018; Published September 07 2018

Citation: Bhattacharyya P, Sengupta S, Bhattacharjee PD, Ganguly D, Dutta D, et al. (2018) Post Exercise Desaturation Can Help Identifying Treatment Responders of COPD Pulmonary Hypertension in Real World: An Appraisal. J Pulm Respir Med 8: 469. doi: 10.4172/2161-105X.1000469

Copyright: @2018 Bhattacharyya P, et al. This is an open-access article distributed under the terms of the Creative Commons Attribution License, which permits unrestricted use, distribution, and reproduction in any medium, provided the original author and source are credited. 
Citation: Bhattacharyya P, Sengupta S, Bhattacharjee PD, Ganguly D, Dutta D, et al. (2018) Post Exercise Desaturation Can Help Identifying Treatment Responders of COPD Pulmonary Hypertension in Real World: An Appraisal. J Pulm Respir Med 8: 469. doi: 10.4172/2161105X.1000469

Page 2 of 5

confirmed subsequently with spirometry according to the criteria laid by the GOLD guideline [7].

\section{Evaluation}

This has been achieved through a defined clinico-radioechocardiographic protocol been used at the institute and been used for publication before $[8,9]$. The protocol demands the presence of at least one point from each of the four headings below:

Clinical symptoms of PH: 1) Shortness of Breath (SOB) either unexplained or disproportionate to clinical or radiological (chest X-ray) findings. 2) Demonstration of desaturation at rest or with mild exercise on pulse oximeter. 3) Other symptoms as unexplained fatigue, syncope, etc.

Chest X-ray: 1) Dilated right main pulmonary artery in PA view. 2) Fullness of the retro-sternal space from right ventricular enlargement in lateral view of chest X-ray. 3) Gross PA dilation/fullness of pulmonary bay.

HRCT chest: 1) Pulmonary artery root diameter $\geq$ aortic root diameter. Pulmonary artery branch diameter $\geq$ accompanying bronchial diameter in 3 or 4 lobes.

Doppler echocardiography: Estimated pulmonary artery systolic pressure $\geq 40 \mathrm{~mm}$ of $\mathrm{Hg}$.

\section{Inclusion of the patients}

A COPD patient with age beyond 45 to 75 years and history of exacerbation in the preceding six weeks, having continuing smoking or uncontrolled co-morbidities (viz. chronic heart failure, chronic renal failure, etc.) or any concomitant lung disease (as tuberculosis, malignancy etc. been apparent on evaluation) that can affect the symptoms, optimal evaluation, and treatment regimen were excluded. Any patient not been compliant to uniform COPD pharmacotherapy and the best possible rehabilitation efforts including home oxygen therapy protocol practiced at the institute were also excluded.

\section{Assessment of health status}

This was done by the measurement of the CAT score at the beginning of the anti-PH treatment and at the follow up visits. CAT score is regarded as a validated assessment for determining the health status for COPD patients. [10]. It had been collected by the same research staff for all the patients.

\section{Determination of exercise response}

This simple exercise test was developed by us at the institute and has been named as 2 Chair Test (2CT) as it includes movement between two chairs.

Two chairs were placed at a distance of 5 feet gap (i.e. end to end) facing each other. A patient is requested to sit on any one of them and rest for a few minutes till his pulse rate and $\mathrm{SpO}_{2}$ remains stable for 30 seconds. This baseline pulse rate and the $\mathrm{SpO}_{2}$ was recorded and the patient was then asked to get up and move to the other chair and sit there before getting up and returning to the previous chair. This is recorded as one movement. Thus, the patient was directed to make five movements at his or her pace otherwise continuously with freedom to stop in between if felt necessary for dyspnoea or any physical discomfort. The test has the provision for premature termination; such performance is noted as $3 / 5$ when the patient could move three movements out of five. The reasons for any premature terminations are noted clearly.

The exclusion criteria for the test includes (a) unwillingness to give a written consent, (b) perceived cardiovascular instability clinicoradiologically or gross left ventricular dysfunction in echocardiography, (c) gross musculoskeletal problem as osteoarthritis of knees, (d) vertigo or neurological disorder affecting normal movement, (e) baseline hypoxemia $\left(\mathrm{SpO}_{2} \leq 90 \%\right)$, and (f) any other systemic problems that can affect the exercise or the performance adversely. Exclusion of Diffused Parenchymal Lung Disease (DPLD) or any other concomitant significant pulmonary disorder were done whenever suspected from clinical examination or chest X-ray through High Resolution Computed Tomography (HRCT) of chest whenever suspected. Similarly, patients with any significant left heart disease on echocardiography were refrained from performing the exercise. After completing the five movements the pulse and saturation were measured immediately and every 10 seconds thereafter for two minutes and the values were recorded. Oxygen supplementation was offered to those who required prolonged time (more than 30 seconds) to recover from de-saturation till the patient turned comfortable.

\section{Selection of patients with COPD-PH for anti-PH treatment}

Any patients having COPD-PH been diagnosed according to the protocol (' $a$ ' and 'b' as above) were included. All of them had similar COPD pharmacotherapy as inhaled corticosteroid with long acting $\beta 2$ agonist plus short acting anti- muscarinic agents according to the protocol. They were then subjected to 2 chair test $(2 \mathrm{CT})$. The patients showing at least $\geq 3 \%$ drop in $\mathrm{SpO}_{2}$ in 2-CT (on the basis of an existing protocol practised by the Institute [9]) were chosen for anti-PH therapy as per the endorsement by the consensus group. The drug prescribed was sildenafil (10 $\mathrm{mg}$ thrice a day to start with and build up to $20 \mathrm{mg}$ thrice daily after a week). Patients with history of intolerance to sildenafil or been on nitrate were excluded. The adverse reactions of the medicine was discussed at the time of prescription (especially of the possibility of increase in dyspnoea, dizziness, blindness, pedal swelling, headache, and uneasiness) and all the patients were requested to stop the drug and report the investigator at the earliest on development of any significant known or suspected adverse reactions. Blanket home oxygen therapy was not prescribed, but all were warned about the chance of emergency demand of oxygen supplementation after the initiation of the anti- $\mathrm{PH}$ therapy and arrange oxygen accordingly.

\section{The follow up protocol}

The follow up was fixed following $90( \pm 15)$ days of initiation and whenever felt necessary by the patient with a mandatory reporting after 7 days of starting medication. At the follow up visits, details of clinical examination with assessment of CAT score and 2-Chair Test were repeated. A repeat echocardiography was also suggested at the follow up visit but it was not made mandatory.

\section{The statistical analysis}

Statistical analysis was done using graph pad prism 7 software. Statistical significance between the two different categories (treatment group and non-treatment group) was analysed using t-test (Wilcoxon t-test). The mean and standard deviation among the two groups were also calculated using the same software.

\section{Results}

A total 147 patients were screened and 89 (60.5\%) patients were selected with the presence of $\mathrm{PH}$. On subjecting the patients to 2-CT, 27 patients showed $\leq 3 \%$ and 62 had $\geq 3 \%$ desaturation of $\mathrm{SpO}_{2}$ qualifying the latter group for the anti- $\mathrm{PH}$ therapy. 12 patients from the treatment group and 8 patients from non-treatment were prescribed oxygen supplementation. The details of both these group of patient from 
Citation: Bhattacharyya P, Sengupta S, Bhattacharjee PD, Ganguly D, Dutta D, et al. (2018) Post Exercise Desaturation Can Help Identifying Treatment Responders of COPD Pulmonary Hypertension in Real World: An Appraisal. J Pulm Respir Med 8: 469. doi: 10.4172/2161105X.1000469

inclusion till the final follow up are presented in Figure 1 and Table 1; it also elaborates the changes in CAT score and 2CT (pulse rate and saturation) statistically between the two groups. Both the groups had similar age, lung function status and mean systolic pulmonary artery pressures. The patients receiving treatment, showed a reduction in desaturation (i.e. from $4.48 \pm 2.9$ to $3.28 \pm 2.4$, p-value $\leq 0.0003$ ) and a CAT score $(12 \pm 5.3$ to $7.2 \pm 3.5$, p-value $\leq 0.0001)$ and improvement on the baseline resting saturation was $(95.91 \pm 2.8$ to $96.25 \pm .5$, $\mathrm{p}$-value $=0.2$ ). When the changes at each 10 seconds were added to see the 'cumulative' value, there has been no difference in the cumulative change of the pulse rate before and after treatment for both the groups. However, while the cumulative change in $\mathrm{SpO}_{2}$ is seen to have reduced in the treated patients significantly (from $4.48 \pm 2.9$ to $3.28 \pm 2.4$; $\mathrm{p}=0.0003$ ), the same has worsened for the untreated group (from 4.66 \pm 2.9 to $5.7 \pm 3.10 ; \mathrm{p}=0.03)$ for the change. In the treatment group1 patient and in the non-treatment 2 patients were reported to be on long term oxygen therapy (LTOT). These changes are displayed by bar chart in Figure 1. The adverse events noted are listed in Table 1.

\section{Discussion}

The results (Table 1) indicate that the baseline pulse rate has dropped in the treated patients with sildenafil while it has increased in the untreated group after 3 months of follow up. However, in the cumulative change in the pulse rate remained nearly unchanged. The impact was best perceived in reduction of desaturation in the treated group $(\mathrm{p}=0.0003)$ and the reverse in the untreated group $(\mathrm{p}=0.03)$. Concomitant to that, the CAT score improved significantly (i.e. reduced) in the treated patients again with the $\mathrm{PH}$ specific agent ( $\mathrm{p}=0.0001)$ suggesting an overall positive effect on the health status. The experience further suggests that the drug sildenafil has been well tolerated in our patients.

$\mathrm{PH}$ develops mostly in the advanced COPD and reasons implicated are hypoxemia, polycythaemia, loss of pulmonary vascular bed from destruction of lung, and hyperinflation $[11,12]$. Hypoxic pulmonary vasoconstriction is considered as a major contributor for the development of PH in COPD and Intestinal lung diseases [13,14]. The development of PH in COPD affects the quality of life and the survival prospect adversely [15]. Hence, the condition needs to be addressed. Incidentally, the $\mathrm{PH}$ guidelines are not clearly instructive about pharmacotherapy of this condition allowing confusion in therapeutic decision making to prevails in this domain [16].

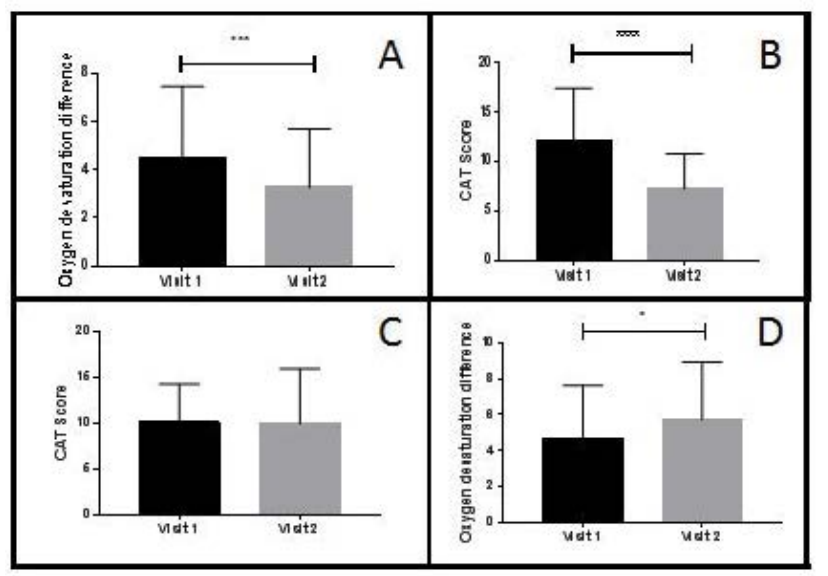

Figure 1: The changes following treatment are represented by bar charts $A$ and C-change in 2CT with or without add-on PH-specific treatment, B and D-change in CAT score with or without add-on PH specific treatment.

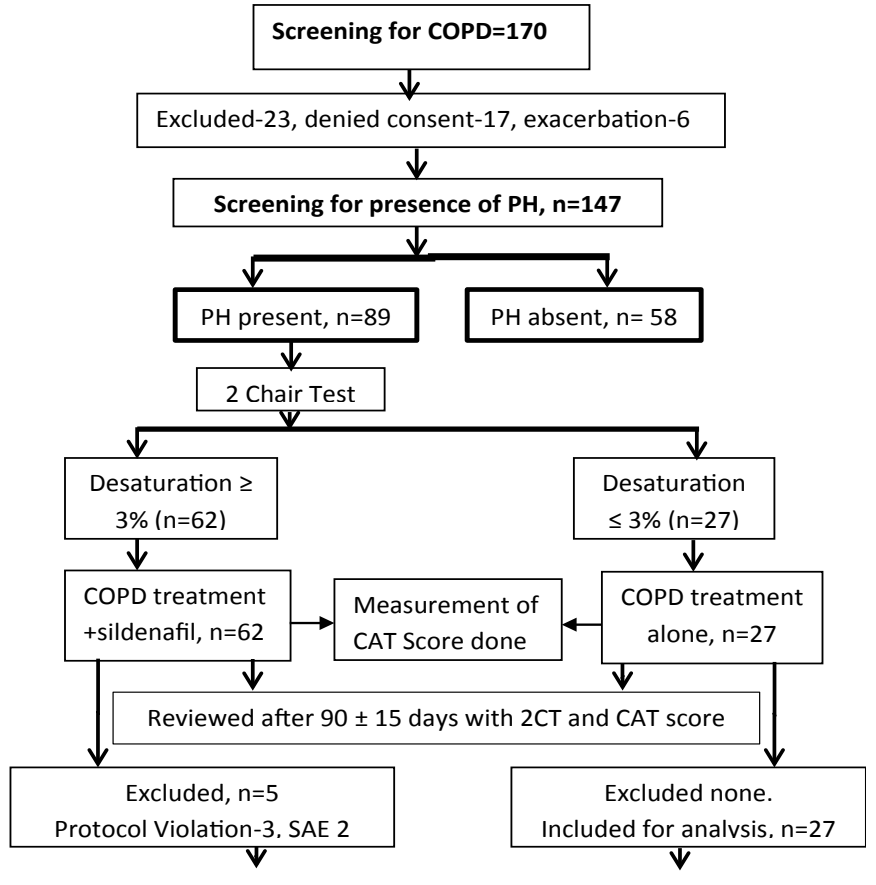

\begin{tabular}{|c|c|c|c|c|c|c|}
\hline \multirow[t]{2}{*}{ Parameters } & \multicolumn{2}{|c|}{$\begin{array}{c}\text { Treatment Group } \\
(n=57)\end{array}$} & \multirow[t]{2}{*}{ P-Values } & \multicolumn{2}{|c|}{$\begin{array}{l}\text { Non-Treatment } \\
\text { Group }(n=27)\end{array}$} & \multirow[t]{2}{*}{ P-Values } \\
\hline & Initial & $\begin{array}{c}\text { After } 3 \\
\text { months }\end{array}$ & & Initial & $\begin{array}{c}\text { After } 3 \\
\text { months }\end{array}$ & \\
\hline M:F ratio & 51:06:00 & \multirow{7}{*}{\multicolumn{2}{|c|}{$\begin{array}{c}\text { No significant } \\
\text { difference observed }\end{array}$}} & 26:01:00 & \multirow{7}{*}{\multicolumn{2}{|c|}{$\begin{array}{c}\text { No significant } \\
\text { difference observed }\end{array}$}} \\
\hline Mean Age & $\begin{array}{c}66.4 \pm \\
6.6\end{array}$ & & & $66 \pm 6.9$ & & \\
\hline Mean (\%) FVC & $\begin{array}{c}59.35 \pm \\
18.7\end{array}$ & & & $\begin{array}{c}58.63 \pm \\
13.9\end{array}$ & & \\
\hline Mean (\%) FEV1 & $\begin{array}{c}43.4 \pm \\
18.9\end{array}$ & & & $\begin{array}{l}41 \pm \\
16.01\end{array}$ & & \\
\hline Mean FEV1/FVC & $\begin{array}{c}51.19 \pm \\
17.1\end{array}$ & & & $\begin{array}{l}\text { P } 52.7 \pm \\
11.16\end{array}$ & & \\
\hline $\begin{array}{c}\text { Mean (\%) FEF25- } \\
75\end{array}$ & $\begin{array}{l}18.6 \pm \\
13.03\end{array}$ & & & $\begin{array}{c}14.77 \pm \\
11.04\end{array}$ & & \\
\hline $\begin{array}{l}\text { Mean PAP } \\
\text { (Systolic) }\end{array}$ & $\begin{array}{l}46.7 \pm \\
8.6\end{array}$ & & & $\begin{array}{c}45.9 \pm \\
15.1\end{array}$ & & \\
\hline Mean CAT & $12 \pm 5.3$ & $7.2 \pm 3.5$ & $<0.0001$ & $\begin{array}{c}10.07 \pm \\
4.15\end{array}$ & $\begin{array}{c}9.92 \pm \\
5.94\end{array}$ & 0.8 \\
\hline $\begin{array}{c}\text { Pulse rate at } \\
\text { baseline }\end{array}$ & $\begin{array}{c}86.48 \pm \\
15.8\end{array}$ & $\begin{array}{c}83.5 \pm \\
12.9\end{array}$ & 0.13 & $\begin{array}{c}84.66 \pm \\
17.08\end{array}$ & $\begin{array}{c}86 \pm \\
13.23\end{array}$ & 0.3 \\
\hline $\mathrm{SaO}_{2}$ at baseline & $\begin{array}{c}95.91 \pm \\
2.8\end{array}$ & $\begin{array}{c}96.25 \pm \\
2.5\end{array}$ & 0.2 & $\begin{array}{c}95.25 \pm \\
2.1\end{array}$ & $\begin{array}{l}94.9 \pm \\
2.7\end{array}$ & 0.6 \\
\hline $\begin{array}{c}\text { Maximum } \\
\text { change in PR }\end{array}$ & $\begin{array}{c}20.3 \pm \\
9.3\end{array}$ & $\begin{array}{c}20.9 \pm \\
9.5\end{array}$ & NS & $\begin{array}{c}23.14 \pm \\
11.6\end{array}$ & $\begin{array}{c}23.78 \pm \\
9.8\end{array}$ & 0.8 \\
\hline $\begin{array}{c}\text { Maximum } \\
\text { change in } \mathrm{SaO}_{2}\end{array}$ & $\begin{array}{c}4.48 \pm \\
2.9\end{array}$ & $\begin{array}{c}3.28 \pm \\
2.4\end{array}$ & 0.0003 & $\begin{array}{c}4.66 \pm \\
2.9\end{array}$ & $5.7 \pm 3.1$ & 0.03 \\
\hline SAE & \multicolumn{3}{|c|}{ Reported hospitalization-2 } & & & \\
\hline $\begin{array}{l}\text { Adverse } \\
\text { reaction }\end{array}$ & \multicolumn{3}{|c|}{$\begin{array}{l}\text { Increased shortness of } \\
\text { breath-4 }(7.01 \%) \text {, pedal } \\
\text { edema-4 }(7.01 \%) \text {, and } \\
\text { dizziness-2 }(3.5 \%) \text {, and } \\
\text { muscle spasm-2 }(3.5 \%)\end{array}$} & & & \\
\hline
\end{tabular}

Table 1: The flow chart elaborates the details of the patients from screening to final follow up. The table elaborates the baseline characteristics as age, spirometric lung functions of the patients qualifying or not qualifying for anti $\mathrm{PH}$ treatment along with the changes in CAT score and parameters in $2 \mathrm{CT}$ (pulse rate and saturation). SAE: Serious Adverse Event. 
Citation: Bhattacharyya P, Sengupta S, Bhattacharjee PD, Ganguly D, Dutta D, et al. (2018) Post Exercise Desaturation Can Help Identifying Treatment Responders of COPD Pulmonary Hypertension in Real World: An Appraisal. J Pulm Respir Med 8: 469. doi: 10.4172/2161105X.1000469

Page 4 of 5

The knowledge of treatment of any pulmonary hypertension has been borrowed from the treatment of PAH (pulmonary artery hypertension) [15,16]. Physiologically, the PAH and COPD-PH have essential differences. While in COPD-PH there has been a preceding and concomitant compromise of the respiratory reserve there is an isolated reduction in the circulatory reserve in PAH $[17,18]$

The dearth of recommendations for pharmacotherapy of COPD$\mathrm{PH}$, though unfortunate, keeps a space for independent thinking and development. The condition has been addressed with controlled trials [19-24], but the lack of uniformity in selection criteria, choice of the agent, methodology, and the end point selection of the different efforts remains a major concern [19-22,24-26]. Thus, the results have been somewhat conflicting to frame a recommendation for treatment [27]. However, despite that at least two recent meta-analysis have been in favour of treating overt $\mathrm{PH}$ at rest in COPD subjects $[13,28]$

Understanding the status of accessible or exhausted pulmonary and pulmonary vascular reserves appears important to decide an intervention in $\mathrm{PH}$. Since all of our patients belonged to severe COPD (GOLD stage-3 and 4), the chance of improvement of the respiratory reserve was virtually missing in them. Therefore, the very post-exercise desaturation in our 2-Chair test protocol reflects significant exhaustion of the pulmonary vascular reserve. Here, the cardiac output fails to commensurate with the increased oxygen demand from the exercise even though it was a much less rigorous protocol than six minutes' walk test (6MWT). The $3 \%$ fall was empirical and was kept in a way to explain the physiological jeopardy. This understanding had made the $\mathrm{PH}$ group members to contemplate trying pulmonary vasodilatation with sildenafil in a desperate effort to prevent desaturation. Taking into consideration the chance of ventilation-perfusion mismatch, the group decided to build up the dose slowly. The presence of mild increase in dyspnoea as a common side effect suggests that the apprehension was justified and the strategy building up the dose of sildenafil was correct as the dyspnoea could be accommodated without discontinuation of sildenafil. The consensus also decided to observe the patients with less than 3\% desaturation and analyse their trend in 2-CT and CAT score without imposing anti-PH therapy after 3 months. Incidentally, these patients showed a significant worsening in desaturation despite no change in CAT score $(\mathrm{p}=0.03)$. Although the $3 \%$ mark on exercise (2CT) is a reasonable cut-off value to predict the perceptible impact on health status with sildenafil in our COPD-PH patients, it is possible, that some of the patients in the untreated group could have improved had they been given sildenafil. One needs to evolve a more precise medicationstrategy in future for these patients. The $2 \mathrm{CT}$ can measure improvement in pulse rate and saturation and, unlike the 6MWT it does not provide any idea about the exercise capacity. Efforts to establish the role of $2 \mathrm{CT}$ in sick $\mathrm{PH}$ patients as a substitute of six minute walk test may be worthwhile. A better and elaborate measurement of desaturation along with hemodynamic studies may be worthwhile to assess the vascular reserve in patients with ventilatory capacity. We had used a fixed dose of phosphodiesterase- 5 inhibitor sildenafil [29]; it will be interesting to see both the acute and the dose response effects of the agent in future. Other anti-PH drugs too may be tested in the similar fashion in COPD$\mathrm{PH}$ patients. That $\mathrm{PH}$ specific instrument as EmPhasis could have been better for the assessment of the health status [30]; incidentally, we had observed that CAT and EmPhasis correlate well in these patients [31]. The greatest criticism of the work may come from the fact of not looking at the hemodynamic criteria which was difficult to procure in our real world. Similarly lack of the repeat peak pulmonary artery pressure measurement at follow up also remains a serious weakness. Despite them, the improvement in health status (CAT score) parallel to the improvement in desaturation indicate towards the positive impact of our strategy of subject selection and pharmoco-intervention.

\section{Conclusion}

Exercise desaturation guided graded administration of anti-PH medication in COPD-PH patients appears worthwhile. Validation of the exercise test (2CT) and the decision algorithm with hemodynamic data and exercise capacity may prove extremely useful in future to recommend $\mathrm{PH}$ specific therapy in COPD-PH.

\section{Declaration of Interest}

It is declared that none of the authors involved in the work has or had any kind of conflict of interest relating to the research authorities.

\section{References}

1. Rodriguez-Roisin R, Rabe KF, Vestbo J, Vogelmeier C, Agusti A (2017) global initiative for chronic obstructive lung disease (GOLD) $20^{\text {th }}$ anniversary: A brief history of time. Eur Respir J 50: 1700671.

2. Mannino DM, Buist AS (2007) Global burden of COPD: Risk factors prevalence, and future trends. The Lancet 370: 765-773.

3. Lopez AD, Shibuya K, Rao C, Mathers CD, Hansell AL, et al. (2006) Chronic obstructive pulmonary disease: Current burden and future projections. Eur Resp J 27: 397-412.

4. Devine JF (2008) Chronic obstructive pulmonary disease: An overview. Am Health Drug Benefits 1: 34

5. Minai OA, Chaouat A, Adnot S (2010) Pulmonary hypertension in COPD Epidemiology, significance, and management: Pulmonary vascular disease: The global perspective. Chest 137: 39S-51S.

6. Shujaat A, Bajwa AA, Cury JD (2012) Pulmonary hypertension secondary to COPD. Pulm Med: 203952

7. Rabe KF, Hurd S, Anzueto A, Barnes PJ, Buist SA, et al. (2007) Globa strategy for the diagnosis, management, and prevention of chronic obstructive pulmonary disease: GOLD executive summary. Am J Respir Crit Care Med 176: 532-555.

8. Bhattacharyya P, Saha D, Bhattacherjee PD, Das SK, Bhattacharyya PP, et al (2016) Tuberculosis associated pulmonary hypertension: The revelation of a clinical observation. Lung India 33: 135-139.

9. Bhattacherjee PD, Saha D, Das SK, Dey R, Ghosh M, et al. (2014) The Etiological profile and the response to treatment with sildenafil in class III pulmonary hypertension: A brief report. The Pulmoface 14: 5-9.

10. Jones PW, Harding G, Berry P, Wiklund I, Chen WH, et al. (2009) Development and first validation of the COPD Assessment Test. Eur Resp J 34: 648-654.

11. Bhattacharyya $P$, Bhattacharjee $P$, Majhi $S$, Tiwari $P$, Chatterjee $S$ (2014) Role of sildenafil in treating pulmonary hypertension in COPD patients. The Pulmoface 14: 41-45.

12. Jones PW, Harding G, Berry P, Wiklund I, Chen WH, et al. Development and first validation of the COPD assessment test. Eur Resp J 2009 34: 648-654.

13. Chen X, Tang S, Liu K, Li Q, Kong H, et al. (2015) Therapy in stable chronic obstructive pulmonary disease patients with pulmonary hypertension: $A$ systematic review and meta-analysis. J Thoracic Disease 7: 309-319.

14. Weitzenblum E, Chaouat A, Canuet M, Kessler R (2009) Pulmonary hypertension in chronic obstructive pulmonary disease and interstitial lung diseases. Semin Respir Crit Care Med 30: 458-470.

15. Duke HN (1957) Observations on the effects of hypoxia on the pulmonary vascular bed. J Physiol 135: 45-51.

16. Andersen KH, Andersen CB, Gustafsson F, Carlsen J (2017) Pulmonary venous remodeling in COPD-pulmonary hypertension and idiopathic pulmonary arterial hypertension. Pulmo Circ 7: 514-521.

17. Scharf SM, Iqbal M, Keller C, Criner G, Lee S, et al. (2002) Hemodynamic characterization of patients with severe emphysema. Am J Respir Crit Care Med 166: 314-322.

18. Stenmark KR, Fagan KA, Frid MG (2006) Hypoxia-induced pulmonary vascular remodeling: Cellular and molecular mechanisms. Circ Res 99: 675-691. 
Citation: Bhattacharyya P, Sengupta S, Bhattacharjee PD, Ganguly D, Dutta D, et al. (2018) Post Exercise Desaturation Can Help Identifying Treatment Responders of COPD Pulmonary Hypertension in Real World: An Appraisal. J Pulm Respir Med 8: 469. doi: 10.4172/2161105X.1000469

Page 5 of 5

19. Blanco I, Santos S, Gea J, Guell R, Torres F, et al. (2013) Sildenafil to improve respiratory rehabilitation outcomes in COPD: A controlled trial. Eur Resp J 42: 982-992.

20. Lederer DJ, Bartels MN, Schluger NW, Brogan F, Jellen P, et al. (2012) Sildenafil for chronic obstructive pulmonary disease: A randomized crossover trial. COPD 9: 268-275.

21. Rao RS, Singh S, Sharma BB, Agarwal VV, Singh V (2011) Sildenafi improves six-minute walk distance in chronic obstructive pulmonary disease: A randomized, double-blind, placebo-controlled trial. Indian J Chest Dis Allied Sci 53: 81-85.

22. Stolz D, Rasch H, Linka A, Di Valentino M, Meyer A, et al. (2008) A randomized, controlled trial of bosentan in severe COPD. Eur Resp J 32: 619-628.

23. Harris KN, Chalhoub M (2010) The effects of sildenafil in pulmonary hypertension secondary to chronic obstructive pulmonary disease. Am J Respir Crit Care Med 181: A5256.

24. Goudie AR, Lipworth BJ, Hopkinson PJ, Wei L, Struthers AD (2014) Tadalafil in patients with chronic obstructive pulmonary disease: A randomised, doubleblind, parallel-group, placebo-controlled trial. Lancet Respir Med 2: 293-300.

25. Valerio G, Bracciale P, Grazia D'Agostino A (2009) Effect of bosentan upon pulmonary hypertension in chronic obstructive pulmonary disease. Ther Adv Respir Dis 3: 15-21.
26. Rietema H, Holverda S, Bogaard HJ, Marcus JT, Smit HJ, et al. (2008) Sildenafil treatment in COPD does not affect stroke volume or exercise capacity. Eur Resp J 3: 759-764

27. Galiè N, Humbert M, Vachiery JL, Gibbs S, Lang I, et al. (2016) 2015 ESC/ERS guidelines for the diagnosis and treatment of pulmonary hypertension: The joint task force for the diagnosis and treatment of pulmonary hypertension of the european society of cardiology (ESC) and the european respiratory society (ERS): Endorsed by: Association for european paediatric and congenital cardiology (AEPC), international society for heart and lung transplantation (ISHLT). Eur Heart J 37: 67-119.

28. Park J, Song JH, Park DA, Lee JS, Lee SD, et al. (2013) Systematic review and meta-analysis of pulmonary hypertension specific therapy for exercise capacity in chronic obstructive pulmonary disease. J Korean Med Sci 28: 1200-1206.

29. Alp S, Skrygan M, Schmidt WE, Bastian A (2006) Sildenafil improves hemodynamic parameters in COPD- an investigation of six patients. Pulm Pharmacol Ther 19: 386-390.

30. Marallu HG, Sadeghieh-ahari S, Lari SM (2012) The relationship between COPD assessment test (CAT) scores and severity of airflow obstruction in stable COPD patients. Tanaffos 11: 22-26.

31. Sengupta S, Dey D, Bhattacharyya P (2016) Correlating between CAT and emphasis score in group III PH Patients. Lung India 71: 18. 